\title{
Analysis on the Influence Factors of Capacitor Voltage Transformer Dielectric Loss Measurement
}

\author{
Yongdong Li, Qing-da Meng, Po, Yang Zheyuan Zhao, Wei Zhang, Zhuo Pan \\ Jibei Electric Power Maintenance Company, Beijing, China \\ Email: yangpo0416@163.com
}

Received April, 2013

\begin{abstract}
Capacitor voltage transformer (CVT), which is with simple structure, convenient maintenance, functional diversity and high impact pressure strength, is widely used. And its capacitance and dielectric loss Angle measurement is an important test on testing the insulation of the equipment. This paper is mainly to introduce and discuss one of the CVT test methods - the "self excitation method", combined with the actual situation encountered in the work, sums up and expounds the maintenance of CVT and preventive test of influence factors.
\end{abstract}

Keywords: Capacitive Voltage Transformer; Dielectric Loss; the Influencing Factors

\section{Introduction}

In recent years, the capacitive voltage transformer is widely used, because of insulation structure reasonable, high dielectric strength, can make full use of the carrier communication required for the coupling capacitor, in more than $110 \mathrm{kV}$ voltage grade [1].

\section{The CVT Transmission Structure}

Capacitive voltage transformer (CVT) is combined with the capacitive voltage divider, electromagnetic unit (including medium voltage transformer, reactor) and the terminal box. And the wiring principles are shown in Figure 1.

Capacitive voltage transformer, which is set as a converting device between the high tension line and ground, is to drop the system voltage into medium first, and after converting secondary voltage through intermediate transformer, the voltage will be output to the measuring instrument and relay protection device [2].

\section{The Significance and Principle of CVT Dielectric Loss Measurement}

For CVT in operation, its running situation overall is good, however, how to discover the CVT defects, such as the manufacturing quality, water be affected with damp be affected with damp, oil shortage, insulation aging factors and determine the operation state is of great significance to its safe and reliable operation. At present, each capacitance differential pressure unit and the dielectric loss value of CVT was tested by the power out- age method as one of the preventive test project is also the principal means of judging its running status.

According to the structure it is divided into separate loading CVT and Superposition CVT, and the Superposition CVT which has no intermediate extraction voltage terminal is in widely used in China. When this kind of CVT is in field operation, we take the Self-excitation method to measure the partial pressure capacitor of capacitance and dielectric loss instead of the common testing methods [3]. Self-excitation method is based on the middle of the transformer as a testing transformer, excited voltage from the secondary side, Induction of high pressure as power supply for measurement. In recent years,

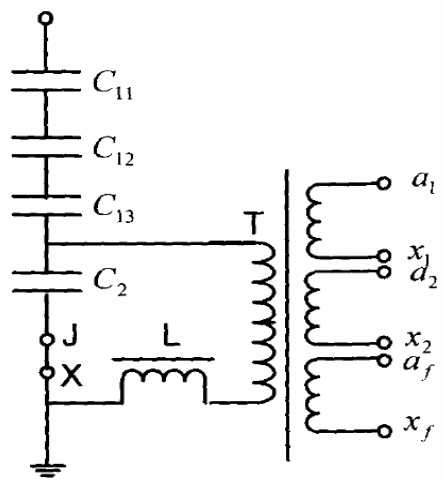

$\mathrm{C}_{11}, \mathrm{C}_{12}$ - coupling capacitance; $\mathrm{C}_{13}, \mathrm{C}_{2}$-partial-voltage capacitance ; $\mathrm{L}$-compensation reactor; $\mathrm{T}$ - medium voltage transformer

Figure 1. The principle diagram of the capacitive voltage transformer. 


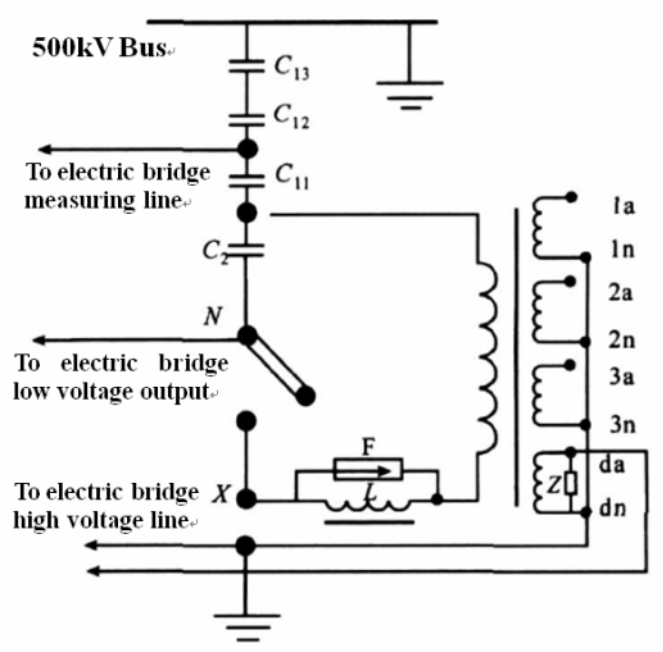

Figure 2. Self-excitation measurement schematic diagram.

the excitation method, which can achieve accurate measurement of the partial pressure of capacitance values and dielectric loss, has been used widely in the CVT routine test at the scene of the substation. Its measuring principle is shown in Figure 2.

In theory any secondary terminals can be used as a self-excited terminal test. But in fact, considering the capacitor voltage effect in the unit test process, we should choose the secondary terminal of large capacity as self-excited terminals to make the higher applied voltage and the test values are more accurate [4]. The capacity of da-dn in secondary terminal is the largest in most of the CVT, and with damping resistance winding, da-dn for the remainder of the experiment can make the process safer, so the self-excitation capacitance measuring pressure method generally choose da-dn terminal is pressurized.

Considering the insulation safety factors in low pressure end, the test voltage should not exceed the end of $\mathrm{C}_{2}$ maximum allowable voltage, $2 \mathrm{kV}$ is selected as a general method of self-excited test voltage in field experiment.

\section{Analysis of Influence Factors of Measurement}

In CVT dielectric loss measurement process, errors sometimes influence the accuracy of the measurement due to some of the factors. The followings analyzed some common influence factors.

\subsection{The Influence of the Temperature}

Temperature has a big influence on the measurement of dielectric loss Angle tan $\delta$, the extent of the impact varies from the different materials, structures [5]. In general, $\tan \delta$ increases with temperature rise. Some insulation material when temperature is below a certain threshold, the $\tan \delta$ may be increased with the decreasing of temperature; And wet material under $0{ }^{\circ} \mathrm{C}$ when water freezes, $\tan \delta$ will be lower. So, measured the insulation of the dielectric loss value in both too high and too low temperature cannot reflect the real situation, but easy to lead the wrong judgment. As a result, the measurement of dielectric loss should be not less than $5^{\circ} \mathrm{C}$.

\subsection{The Influence of the Test Voltage}

In general, at its rated voltage range, tan delta value is almost unchanged. The $\tan \delta$ of good insulation is not significantly increased with the rising of over voltage. But things are different if bubbles, delaminating, shell happens in the insulation [6]. When the test voltage is not enough to make the air bubbles or air gap of the insulation free, the $\tan \delta$ and normal has no obvious difference; When test voltage makes the air in insulation free and produces corona or partial discharge, the tan $\delta$ will be increased as the test voltage increases. Several kinds of typical testing curve is shown in Figure 3.

\subsection{Standard Capacitor}

Capacitance and dielectric loss of the standard capacitor plays an important role in the measurement of CVT by applying self-excitation method. The accuracy of $\mathrm{C}_{2}$ has certain relationship with these parameters since $\mathrm{C}_{n}$ is in series connection with $\mathrm{C}_{1}$. For example, when a CVT (Model TYD110/ $\sqrt{3}-0.01 \mathrm{H}$, Nominal voltage divider $\mathrm{C}_{1}=12500 \mathrm{pF}, \mathrm{C}_{2}=50000 \mathrm{pF}$, dielectric loss $\tan \delta=$ $0.1 \%$ ) is tested with $\mathrm{Cn}=100 \mathrm{pF}, \mathrm{C}_{1}$ equivalent to series connection of a resister and a capacitor [7].

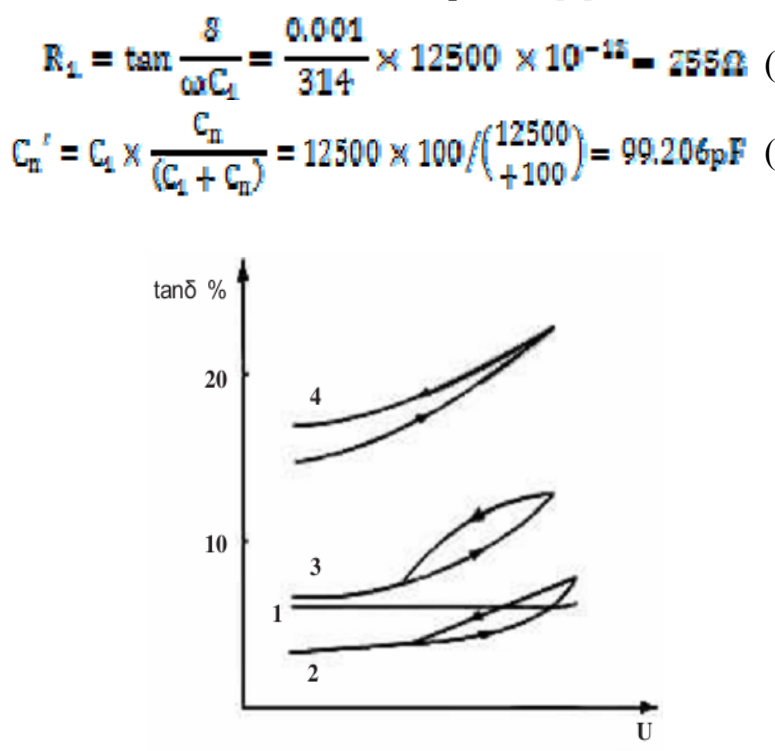

1- good insulation; 2 - when the insulation aging; 3 - there is air gap in insulation; 4 - insulation be affected with damp be affected with damp

Figure 3. Tan delta and the curve of voltage. 
The error of the standard capacitor becomes:

$$
(99.206-100) / 100=-0.79 \%
$$

And the equivalent dielectric loss is:

$$
\begin{aligned}
\tan z^{*}=\operatorname{RoC}_{\mathrm{n}}{ }^{*} & =255 \times 314 \times 99206 \times 10^{-12} \\
& =7,9 \times 10^{-6}
\end{aligned}
$$

As is shown above, the measurement error is negligible as long as the standard capacitor is properly opted, i.e. capacitance high and dielectric loss low, or the accuracy will be affected.

\subsection{Insulation of the Low Voltage End}

As a result of insulation degradation of the terminal block on the low voltage end of the voltage divider, the measured dielectric loss of $\mathrm{C}_{1}$ may exceed its true value by applying self-excitation method. Since terminal $\mathrm{N}$ has a relatively high potential about $2 \mathrm{kV}$ in testing, leakage current upon the minor bushing and terminal block will lower down the accuracy due to the degradation of insulation, which is commonly caused by damp conditions of the secondary terminal box. In order to get the true value, measurements can be put out after drying process.

In the case of $\mathrm{C}_{2}$ testing, the main influencing factor lies in the method of measurement, since terminal $\mathrm{N}$ has a relatively low potential and directly connects to the testing bridge. The error is negligible and the testing result makes approximately its true value.

Many factors will have influence on the testing result by applying self-excitation method, yet the measurement value of $\mathrm{C}_{2}$ well indicates the real situation of the voltage divider, as it shares the same insulator and the corresponding dielectric loss are of the same, while the higher value of $\mathrm{C}_{1}$ represents the damp conditions of secondary terminal and minor insulator.

\subsection{Other Factors}

The voltage of PT components interfere the voltage phase and amplitude of terminal $\mathrm{N}$ through the distributed capacitance coupling, thus affect the measurement accuracy [8].

Electric filed in the vicinity caused by other operating equipments also influence the testing result. To eliminate the interference, frequency conversion method is often applied in field testing.

\section{Conclusions}

As is discussed, the accuracy in the measurement of CVT dielectric loss is influenced by various factors, i.e. ambient temperature, testing voltage, standard capacitor and the insulation status of low voltage end, the last two of which have greater impact on the testing result and are commonly emerged in field. When the measurement value exceeds its true value, further analysis should be carried out before judgment so as to ensure the stable operation of power grid.

\section{REFERENCES}

[1] T. X. Chen, Y. Z. Wang and S. J. Hai, "Electrical Test," China Electric Power Press, Beijing, 2008.

[2] W. He, "Measurement of Tangent Loss in Capacitor Voltage Transformer," Northwest China Electric Power, Vol. 31, No. 5, 2003.

[3] W. L. Yang, H. Q. He and H. S. Wang, "Impacts of CVT Damped Secondary Outlet Block on Dielectric Loss Measurement by Self-Excitation Method," East China Electric Power, Vol. 39, No. 9, 2011.

[4] T. Li, X. P. Du and H. G. Liu, "Discussion about Self-excited Method Error on Capacitive Voltage Transformer," Power System Protection and Control, Vol. 37, No. 5, 2009.

[5] Q. Rao, "Discussion about Measurement on 110-220 kV Capacitive Potential Transformer," Guangxi Electrical Engineering, 2006.

[6] Z. M. Liang and Y. M. Tan, "Tan $\delta$ Test and Analysis for CVT's EM Unit," High Voltage Engineering, 2006.

[7] X.D. Jin and K. Y. Jing, "Self-excited Method for Superposition CVT," Jiangsu Electrical Engineering, 2005, Vol.11.

[8] Y. G. Yue, J. B. Yin and Y. P. Wang, "Analysis on 500 $\mathrm{kV}$ Capacitor Voltage Transformer Site Testing By Self-excitation Method," Power Capacitor \& Reactive Power Compensation, Vol. 31, No. 4, 2010. 